Math. Model. Nat. Phenom.

Vol. 7, No. 3, 2012, pp. 186-203

DOI: $10.1051 / \mathrm{mmnp} / 20127312$

\title{
Mathematical and Computational Models in Tumor Immunology
}

\author{
F. Pappalardo ${ }^{1}$, A. Palladini ${ }^{1}$, M. Pennisi, F. Castiglione ${ }^{3}$, S. Motta* \\ ${ }^{1}$ Università degli Studi di Catania, Catania, Italy \\ ${ }^{2}$ Cancer Research Section, Department of Experimental Pathology \\ University of Bologna, Bologna, Italy \\ ${ }^{3}$ Institute for Computing Applications "M. Picone", National Research Council of Italy \\ Viale Manzoni 30, 00185 Rome, Italy
}

\begin{abstract}
The immune system is able to protect the host from tumor onset, and immune deficiencies are accompanied by an increased risk of cancer. Immunology is one of the fields in biology where the role of computational and mathematical modeling and analysis were recognized the earliest, beginning from 60s of the last century. We introduce the two most common methods in simulating the competition among the immune system, cancers and tumor immunology strategies: differential equations and rule-based models. Several specific implementations are presented, describing in details how they work and how they advance or contribute the field of tumor immunology.
\end{abstract}

Keywords and phrases: cancers, tumor immunology, agent based models, mathematical modeling, vaccines

Mathematics Subject Classification: 81T80, 92B05, 46N60, 62P10, 92C60

\section{Introduction}

The immune system, along with the central nervous system, represents the most complex biological system in nature: the only way to get a chance to understand it is the use of several different research strategies. Recently it has been demonstrated that the immune system plays an essential role in cancer dynamics $[41,65,75]$.

Conventional cancer therapy usually has severe side effects. For this reason, strategies to induce an efficient and effective immune system response against cancer cells, have received an increasing research efforts [28,57]. Vaccines [19,63], monoclonal antibodies [76], lymphocytes [31,32,35], or cytokines [29,42], represent the main stream of tumor immunology methods that are presently investigated for this target.

In general, complex systems can be analyzed through several mathematical and computational approaches. Biological systems represent complex systems and then they are suitable to be treated with modeling methodologies.

\footnotetext{
${ }^{*}$ Corresponding author. E-mail: motta@dmi.unict.it
} 
In this work, we introduce the two most common methods in simulating the competition among the immune system, cancers and tumor immunology strategies: differential equations and rule-based models. The paper is organized as follows. In 2 we briefly introduce the immune system and its main functions. In Section 3 we provide a primer in tumor immunology. Sections 4 and 5 are devoted to present several mathematical and computational approaches. Finally in 6 we draw our conclusions.

\section{The immune system}

The immune system protects organism from infectious diseases, reacting to components of microbes (virus, bacteria, parasites) recognized as foreign. The host defense combines innate (or natural) and adaptive immune responses, two arms of an integrated system in which cells and molecules act cooperatively [1].

A first line of defense against microbes is provided by innate immunity. This first response is essentially the same for repeated infections. Recognition of structures common to groups of related microbes is mediated by cellular receptors referred as pattern-recognition receptors. The main components of innate immunity are physical barriers, soluble mediators and specialized killer cells.

Pattern-recognition receptors include three receptors families: Toll-like receptors recognize bacterial and viral nucleic acids, flagellin, bacterial peptides, lipopolysaccharide and other bacterial components; Mannose receptors bind carbohydrate moieties on several pathogens; Seven-transmembrane spanning receptors are activated by bacterial peptides or by endogenous chemokines.

Physical barriers include epithelial surfaces between foreign agents and host such as skin and mucosal surface of the gastrointestinal and respiratory tracts. Another component of this group is the blood coagulation system that forms a protective cloth over wounds.

The host organism produces many soluble mediators able to inhibit viral infections and kill bacteria. This class includes natural antibiotics such as defensins, enzymes for bacterial lysis, complement molecules, opsonins to promote phagocytosis, and cytokines for the induction of an anti-microbial state in neighboring cells.

The specialized cellular effectors of innate immunity migrate towards microbes and kill them by phagocytosis (granulocytes and macrophages) or by releasing of lytic substances in the environment (natural killer -NK- cells). Granulocytes, containing lytic substances packed in intracellular granules, include neuthrophils, against bacteria, and basophils, against parasites. Circulating monocytes differentiating to macrophages are placed in all tissues and organs. Macrophages survive much longer than granulocytes at sites of injury and so are the main late cellular effectors of the innate response. Natural killer cells closely resemble lymphocytes morphology. NK cells kill infected cells both directly by releasing of perforins and granzymes granules and indirectly by macrophages activation.

The second line of defense against infections is provided by adaptive immunity that specifically recognizes distinct components (antigens) and stores the information on the acquired experience against these components to improve the immune response to repeated exposures. Lymphocytes are the actors of this immune response that is specific for foreign antigens and tolerant for autologous (self) components.

Lymphocytes circulate in blood, tissues and in lymphatic vessels, moreover reside in specialized organs (lymphoid organs, which include the thymus, the spleen and lymph nodes). Lymphocyte population is composed of millions of clones with different clonotypic specific antigen receptors able to recognize a high number of different antigens (in the order of $10^{20}$ ). The enormous variety of lymphocytes receptors is due to a unique DNA rearrangement of these molecules, peculiar of lymphocytes. Specifically, receptor genes comprise two or three sets of $10^{1}-10^{2}$ alternative segments encoding the variable antigen-binding part (V , D and J segments) region, separated by non-coding DNA segments and followed by one or few segments encoding the constant $(\mathrm{C})$ part of the receptor. One segment from each variable set is chosen at random and put near to one another by enzymes, which cause also sequence alterations at the point of contact between the various segments. Finally, variability is increased by the dimerization of receptor molecules encoded by separate genes, each undergoing DNA rearrangement independently. The association of two 
receptor chains is necessary for a functional membrane antigen receptor. At the end of the process the rearranged DNA of lymphocytes is different from that of all other cells of the organism.

Lymphocytes population includes cells with different types of antigen receptors. The main distinction is between $\mathrm{T}$ and $\mathrm{B}$ cells.

Immunoglobulins (Ig) are membrane antigen receptors of B cells. Antigen-stimulated B cells differentiate into plasma cells which actively secrete antibody, a soluble form of the receptor. Antibodies are classified in different isotypes: IgM, the first antibodies produced in primary immune response; IgG, the main class of antibodies produced in secondary immune responses and released in the bloodstream; IgA, mainly secreted in milk, tears, saliva and intestinal juices; IgE, related to allergies. Antibodies bind to their cognate antigens yielding insoluble antigen-antibody complex rapidly removed from the blood. Moreover antibodies can bind to cell membranes activating complement components that can lyse the bound cell. The antigen-antibody binding elicits immune responses (phagocytosis and cell lysis) of leukocytes expressing surface receptors for the constant part of antibodies.

TCR is the surface antigen receptor of T cells. TCR activates a signaling cascade by recognizing of small peptides presented on the cell membrane bound to a cellular protein called the major histocompatibility complex (MHC). T cells include three main subpopulations with different functions: cytotoxic T cells (Tc, CTL, CD8+) directly kill cells expressing the antigen; regulatory T cells (Treg) inhibit immune responses; helper T cells (Th, CD4+) positively regulate the activity of B and Tc cells. Th cells are further divided into type 1 and type 2 cells (Th1 and Th2). Th1 cells release $\gamma$-interferon (IFN- $\gamma$ ) and other cytokines to stimulate immune responses directed against viruses and intracellular bacteria, whereas Th2 cells release interleukin 4 (IL-4) to stimulate the immune response against parasites.

Different cells are needed to produce an adaptive immune response. Foreign proteins, captured and processed by specialized leukocytes (antigen presenting cells-APC-), are presented to Th cells in association with MHC molecules. Recognition of the peptide-MHC complex by a specific TCR activates the Th cell that proliferates and secretes cytokines, in turn activating antibody production by B cells and replication of Tc cells. Antibodies, Th and Tc cells reach the periphery where they encounter free pathogens and infected cells. Antibodies can inactivate for example free viruses by direct binding and kill virus-infected cells through complement-mediated cytotoxicity and, with the help of NK cells, antibodydependent cell citotoxicity (ADCC). Th cells secrete an array of cytokines that attract other leukocytes, inhibit microbes replication, and stimulate hemopoiesis to increase the number of leukocytes in the body. Tc cells recognize and kill infected cells expressing specific peptide-MHC complexes on their membrane. The first time that a lymphocyte recognizes a given antigen (primary immune response) leaves behind a population of memory cells that will respond more promptly and more efficiently to subsequent encounters (secondary immune response). Only the clones expressing receptors for a specific antigen are activated upon infection, while all other clones remain inactive.

The presence of random events in the generation of specific antigen receptors implies the risk of producing autoreactive receptors. To prevent autoimmunity, the differentiation of lymphocytes is accompanied by selective mechanisms that ensure tolerance to autologous (self) components through the destruction or the inactivation of autoreactive clones. T cells produced by hemopoiesis in the bone marrow migrate to the thymus where $\mathrm{T}$ cell receptor rearrangement takes place followed by clearing of clones expressing inappropriate receptors (clonal deletion). T cells are exposed to a comprehensive sampling of the peptides that they will subsequently encounter in the periphery. Autoreactive $\mathrm{T}$ cells that recognize autologous MHC-peptide complexes die by apoptosis (negative selection), as do T cells with missing or defective TCR that completely fails to interact with autologous MHC. Only T cells whose TCR interacts with appropriate affinity with the $\mathrm{MHC}$, thus potentially reactive with foreign antigens, survive and thrive (positive selection). Central (thymic) tolerance is not completely efficient, therefore all normal adults harbor autoreactive $\mathrm{T}$ cells that are rendered harmless by tolerogenic processes in the periphery of the body because if a $\mathrm{T}$ cell encounters the antigen on cells lacking costimulatory molecules the ability of the $\mathrm{T}$ cell to be activated is inhibited (anergy). 
Finally, malfunctions of immune system cause pathological diseases such as autoimmune diseases (break down of tolerance), allergy (hypersensitivity to specific antigens contained in food, dust, pollens, animal components, chemical products and drugs), primary immunodeficiency (genetic defects), secondary immunodeficiency (HIV infections or drug treatments), organ rejection (tissue incompatibility).

\section{Tumor immunology}

The immune system is able to protect the host from tumor onset, and immune deficiencies are accompanied by an increased risk of cancer $[18,20]$. This theory of cancer immune surveillance, postulated in the 1960 s, was confirmed by study of genetically-modified mice (GEMs) bearing genetically-immune defects. Immunodepressed mice are more prone to tumor onset than wild-type mice. Also in humans the incidence of various tumor types (lymphomas, leukemias, melanomas, virus-related tumors) is significantly increased in immunodeficient individuals as, for example, patients with AIDS or people treated with immunosuppressive drugs. Finally, the immune system physiologically prevents tumor onset as showed by animals models and human studies.

The immune surveillance of tumors is not $100 \%$ effective. Tumors arise in hosts which a severe and stable immune deficiency. Moreover temporary immunodepression permits the tumor to progress. Tumors eventually arise because immune system becomes less efficient with aging. Cancer cells could also acquire the capability to exploit immunological mechanisms and improve the immune surveillance evasion: incipient tumors can take advantage of an inflammatory environment; the increasing genetic instability of precancerous cells favours the emergence of clones of different immunogenicity. The poorly immunogenic ones are favourite to evade immune system tumor recognition. Finally, tumors can acquire the ability to release factors and cytokines to subvert normal immune reaction mechanisms [12]. Immune responses against tumors have been depthly studied and include various cells and molecules of innate and adaptive systems.

Phagocytic cells (granulocytes, macrophages) directly destroy tumor cells and induce cell degradation producing cell fragments that can be picked up by antigen presenting cells. Dendritic cells are professional APCs and load tumor antigens in the periphery then migrate to lymph nodes. NK cells kill tumor cells with a low MHC expression and play a key role in the defense against circulating metastatic cells. Innate immune response against tumor includes also myeloid suppressors cells that can inhibit antitumor immune response. The adaptive immune response against tumors is sustained by T cells. After antigen presentation Th cells proliferate and elicit, by cytokines secretion, Tc-killing of tumor cells. Also cytokines can directly affect cancer cells. Finally Treg cells can inhibit antitumor response.

$\mathrm{B}$ cells and antibodies are considered generally marginal. One reason is that most of solid tumors are protected from lysis by antibodies and by complement. Moreover B cells can even downregulate $\mathrm{T}$ cell responses and facilitate tumor growth. Although $\mathrm{T}$ cells are often considered the major antitumor mechanism activated by immune system, hundreds of different approaches clinically tested over the years have disappointed the expectations of effective therapies for oncologic patients [66]. The development of monoclonal antibodies against cell surface molecules led to clinical success in patients with lymphomas and also with breast cancer and other solid tumors. So antibodies could be truly actors of new induced immune responses against cancers. Tumor antigens include a wide set of tumor-associated molecules recognized by the immune system of the host [49]. Tumor antigens can be divided into four major classes: cancer testis antigens or melanoma testis antigens, a class of previously unknown molecules expressed during normal gametogenesis and by various tumor types, in particular melanoma; differentiation antigens, expressed during cell differentiation of a given lineage and by tumor cells of the same lineage; "shared" tumor antigens expressed by various tumor types; "unique" tumor antigens, products of point mutations or chromosomal translocations in oncogenes or tumor suppressor genes. Summarizing, tumor antigens are expressed by normal, non-neoplastic cells. Consequently a successful antitumor immune response against such self antigens requires a break of immune tolerance.

The study of tumor immunology has led to the development of approaches to further stimulate antitumor immunity. Immunological strategies for the cure of established tumor masses (immunotherapy) 
have given poor results suggesting that successful antitumor strategies should be addressed to adequately stimulate immune system before tumor onset (immunoprevention), to protect the organism from specific cancers, and so that prevention is more effective than cure in the tumor immunology field [37].

A logical cancer immunopreventive approach consists in prevention of tumors related to infectious agents, such as human hepatitis B and C viruses (HBV and HCV) and human papilloma virus (HPV), closely related to liver and cervical carcinoma respectively. In fact, vaccination against HBV has reduced the incidence of post-hepatitis hepatocellular carcinoma (HCC) and an effective vaccine against $\mathrm{HCV}$ infection is needed to complete the immunoprevention of post-hepatitis HCC. Vaccines against HPV are expected to greatly reduce the incidence of cervical carcinoma. A less obvious approach deals with the immunoprevention of non-infectious tumors. In the last decades preclinical studies have produced good results showing that immunological approaches effectively limit tumor development. Treatments include non antigenic-specific strategies (IL-12, alpha-galactosyl ceramide, Treg depletion, and others) that enhance a broad immune reactivity including antitumor components, and antigenic specific approaches (passive as monoclonal antibodies or active as vaccines). Active immunoprevention effectively reduced or delayed carcinogenesis in a variety of different model systems although it is still far from the clinic and several questions need to be answered before progressing into clinical trials [38].

The choice of a right target is an important issue to develop an effective vaccine. Up to now many tumor antigens have been described, but only a few molecules proved to be good target antigens for cancer immunoprevention. Tumor associated molecules that are essential for tumor growth and progression could be suitable cancer vaccine targets, since they cannot be easily downmodulated or negatively selected in precancerous lesions under the pressure of a specific immune attack. Lollini and colleagues have defined these molecules as oncoantigens [37]. Oncoantigens should be poorly expressed by normal adult tissues, but overexpressed by tumor [11]. Moreover the presence of target antigens on the cell membrane seems to be an optimal condition to obtain both a cell-mediated and an antibody-mediated immune response. Recently the definition of "oncoantigen" was extended to extracellular secreted molecules (oncoantigenclass II) and intracellular molecules (oncoantigen-class III) [38].

Vaccines consisting of live, irradiated or genetically modified tumor cells, dendritic cells, proteins, peptides or naked DNA have been tested in GEMs. The most successful vaccines in preclinical studies consisted of combinations of the target tumor antigen with other immunological signals acting as adjuvants, such as microbial CpG sequences, cytokines, allogeneic glycoproteins of the major histocompatibility complex (MHC) and/or co-stimulatory molecules. On the whole the efficacy of a tumor preventive vaccine depends both on a high level of the antigen and on the adjuvants. Tumor antigens, both in humans and GEMs, are mostly self-antigens overexpressed by tumor. Adjuvant molecules, inducing an aspecific immune response, contribute to break the immune tolerance to tumor antigens. Therefore the efficacy of a vaccine is based on its ability to reverse a tolerant state and activate low-avidity immune reaction mechanisms that escape the central tolerance [37].

Concerning the immune mechanisms induced by immunopreventive vaccines, a coordinate induction of T-helper cells, cytotoxic T lymphocytes and, above all, antibodies seems to determine vaccine protective ability in preclinical models. In order to ensure a long-term immune response, vaccines should be able to elicit immune memory mechanisms that are believed to persist for a very long period. This could only be possible by repeated boost vaccinations.

Among target tumor antigens for cancer immunopreventive strategies the human epidermal growth factor receptor 2 (referred to as HER-2 or ErbB2), a surface molecule, is one of the widely studied. This receptor can lead to neoplastic transformation and it is overexpressed in 25-30\% of human breast cancers [69]. Cancer immunopreventive studies in rat HER-2/neu transgenic mice have produced a good number of approaches able to delay and/or reduce tumor onset up to a complete protection from tumor carcinogenesis driven by HER-2/neu oncoantigen [38]. HER-2/neu transgenic mice are likely the most extensively studied models for the evaluation of immunopreventive approaches against mammary cancer. These models, developed in the last 20 years, comprise various transgenic mouse lines differing in the 
genetic background (FVB, BALB/c, CD-1), the type of promoter, the type of oncogene and the species of origin [74].

Specifically, HER-2/neu transgenic mice (BALBneuT) have been used for our cancer immunopreventive studies. BALBneuT mice carry the rat neu activated oncogene under the control of the MMTV promoter. Female mice develop multifocal mammary carcinomas, resembling the main aspects of human counterpart, with a short latency, about 20 weeks of age. Within 33 weeks of age lobular carcinomas are palpable in all 10 mammary glands [9].

One of the most preclinical effective vaccines is Triplex cellular vaccine, set up in our laboratory, that combines multiple immune signals in the same vaccine: the target antigen with two "adjuvant" stimuli, IL-12 and allogeneic MHC molecules. The main purpose of IL-12 is to enhance antigen presentation and Th cell activation in response to the antigen. Allogeneic MHC molecules stimulate multiple T cell clones and cause a broad production of immunostimulatory cytokines that amplify immune responses $[17,45]$. Repeated administrations of the Triplex vaccine to young BALBneuT mice resulted in a complete block of mammary carcinogenesis. Almost all vaccinated mice remained tumor-free at one year of age, whereas all untreated mice had multiple mammary carcinomas at six months of age. The efficacy of Triplex vaccine was related to the number and distribution of administrations along the mouse life. Protective effectiveness of Triplex is provided by frequent vaccinations in the first weeks of treatment and periodic boosts long life [51].

The main actors of the immune response elicited by the Triplex vaccine were IFN- $\gamma$ and anti-HER$2 /$ neu antibodies. IFN- $\gamma$, produced by Th1 lymphocytes and Tc lymphocytes, through IL-12 induction, promotes the isotypic switch to Th1-type immunoglobulins G (IgG). Moreover IFN- $\gamma$ causes block of cell proliferation and apoptotic cell death. The killing of HER-2/neu positive mammary carcinoma cells is mediated by classical immune mechanisms as well as by downmodulating the HER-2/neu receptor. Moreover IFN- $\gamma$ can induce production of antiangiogenic factors. Anti-HER-2/neu antibodies, specifically IgG2a and IgG2b murine subclasses, showed antitumor activities mediated both by immune mechanisms, such as ADCC, and by induction of HER-2/neu recycling and inhibition of receptor dimerization $[17,45]$. The importance of IFN- $\gamma$ and anti-HER-2/neu antibodies was confirmed by studies in BALBneuT mice lacking antibodies or IFN- $\gamma$ production. In these mice Triplex vaccine loss the ability to prevent mammary carcinoma onset [46].

Moreover recently we showed that vaccine efficacy was provided by a rapid increase of anti-HER-2/neu antibodies in the first phases of vaccination and the maintenance of an high level along life. Finally, elder mice reacted less efficiently to Triplex vaccine than younger ones so in elder mice more frequent boosts are required [51].

Preclinical immunopreventive studies did not show evidence of significant adverse effects. However tumor cells oncoantigens are cross-reactive with normal cells and the possibility of harmful side effect in clinics must be taken into account. The risk might increase if cancer vaccines are combined with Treg depletion or inactivation treatment.

Finally cancer preventive vaccines that fail to curative purposes can be effective in the therapy of metastases [47] and so potentially effective in adjuvant therapy in clinics.

\section{Mathematical models}

A mathematical model uses mathematical language, such as formulas to describe a system. An early idea of such modeling of the immune system is from Sercarz and Coons [67]. The idea was modified by Sterzl [72] and led to the construction of mathematical models of the immune response [6,24]. Based on these mathematical models many contemporary models were built using differential equations.

Differential equation based models of the immune system have been recognized as efficient models producing good simulation results [39,62]. They have been very popular, and a wide range of immunological phenomena have successfully been simulated using differential equation based models. The behavior of the model is then compared to data obtained by in vivo or in vitro experiments, and if in agreement, the model can be used for description of immune function as well as for prediction of its behavior. 
Differential equation based models are still used widely for simulating many different phenomena, including the modeling of immune system related pathologies and in tumor immunology. For example, Ha Youn Lee et al. [33] developed a model which represented published experimental findings on primary influenza virus infection. Their model was used to explore alternative structures for interactions between virus and immune cells, for example, comparing virus kinetics when antigen delivery and immune cell priming occurred through direct interaction of virus and immune cells or through a cellular intermediate.

In [22] the authors presented a novel inverse problem technique to construct a basic mathematical model of the interacting populations at the tumor-host interface, a highly complex, dynamical structure dominated by nonlinear processes for which there is no clear theoretical framework of understanding. They provided a simple mathematical framework which encompasses the critical behavior of this interface, namely, the growth of tumor tissue into the surrounding host tissue, elucidating key biological parameters controlling this behavior.

Tumor growth represents an important issue in modeling tumor immunology. In [2] a physiologically structured lattice model for vascular tumor growth is presented. It takes into accounts blood flow and structural adaptation of the vasculature, transport of oxygen, interaction between cancerous and normal tissue, cell division, apoptosis, vascular endothelial growth factor release, and the coupling between these processes.

Mathematical kinetic theory of active particles can be applied to the modelling of the very early stage of cancer phenomena, specifically mutations, onset, progression of cancer cells, and their competition with the immune system. In [7], the authors present a review that explains in details the multiple ways to apply this approach.

Analytical techniques allow modelers to define the system behavior and their associated parameters and initial conditions. In [8] the author presented a mathematical model formulated by a nonlinear delayed differential equation. The model was used for investigating the effect of variations in virus and cytotoxic T-cells response parameters on LCMV infection outcome and suggesting predictions for experimental studies, in particular the phenotype of LCMV-WE infection in C57BL/6 as a function of initial virus doses. Modifications, such as adding time delays or age-structured partial differential equations [3] make these models more faithful representations of biological reality. In Anitia's work, two models describing the CD8 responses by a partial differential equation or a system of ordinary differential equations were reported. Both of these models showed high consistency with the experimental observations.

In conclusion, differential equation models capture the general behaviour of system and the global parameters of the model can be easily adjusted. However, these models are limited to a specific observable function, and they do not capture the complex interplay of various factors influencing observed behaviour. This limitation prevents their application for studies of the immune system as a complex system. A more detailed review of earlier applications of differential equations for modeling the immune system can be found in $[39,61]$.

\section{Mathematical modeling of artificial immunity against mammary carcinoma}

SimTriplex [52], one of the core simulator of the EC-funded ImmunoGrid project [23,54], is a specialized cellular automaton in modeling mammary carcinoma, Triplex vaccine and the immune system competition based on a modification of Celada-Seiden framework. It will be detailed in Section 5

The main goal of the SimTriplex model was to answer to the question whether the Chronic protocol is the minimal vaccination protocol yielding complete protection from tumor onset. The Chronic schedule comprised four vaccination over the first two weeks of each 4-week cycle (19) up to the 63rd week of age (60 vaccinations). However computational models do not easily allow an asymptotic analysis. For this reason an Ordinary Differential Equation (ODE) model that has been built upon the same conceptual scheme used for the computational model is under development. The preliminary model presented in [59] is composed by the following 12 equations:

$$
\frac{d V C}{d t}=k_{i n}(t)-\left(\mu_{4}+\alpha_{1} T C+\alpha_{2} A b+\alpha_{3} N K\right) \cdot V C
$$




$$
\begin{aligned}
& \frac{d T A A v}{d t}=\alpha_{10}\left(\mu_{4}+\alpha_{1} T C+\alpha_{2} A b+\alpha_{3} N K\right) \cdot V C+ \\
& -\left(\mu_{10}+\mu_{11} B+\alpha_{14} A b+\mu_{15} M P+\mu_{16} D C\right) \cdot T A A v \\
& \frac{d M P}{d t}=\alpha_{15} T A A v+\alpha_{16} T A A c-\mu_{17} M P \\
& \frac{d D C}{d t}=\alpha_{17} T A A v+\alpha_{18} T A A c-\mu_{18} D C \\
& \frac{d B}{d t}=\alpha_{20} T H+\left(\alpha_{21} I L 2-\mu_{20}\right) \cdot B \\
& \frac{d T H}{d t}=\alpha_{22} T A A v+\alpha_{23} T A A c+\alpha_{24} V C+\left(-\mu_{21}+\alpha_{25} I L 2+\alpha_{26} I L 12\right) \cdot T H \\
& \frac{d I L 12}{d t}=\alpha_{75} \cdot k_{i n}(t)-\left(\mu_{5}+\alpha_{27} T H+\alpha_{28} T C+\alpha_{29} N K\right) \cdot I L 12 \\
& \frac{d I L 2}{d t}=\alpha_{30} T H-\left(\mu_{30}+\alpha_{31} B+\alpha_{32} T C\right) \cdot I L 2 \\
& \frac{d C C}{d t}=\left[\left(1-\frac{C C}{c_{\max }}\right)\right] \cdot\left[k_{1} C C\right]+p_{1}-\left(k_{2} N K+k_{3} T C+k_{4} A b\right) \cdot C C \\
& \frac{d T C}{d t}=\alpha_{40} \cdot V C+\left(\alpha_{41}-\mu_{40}\right) \cdot T C \\
& \frac{d T A A c}{d t}=\alpha_{50}\left(k_{2} N K+k_{3} T C+k_{4} A b\right) \cdot C C-(\mu 50+ \\
& \left.+\mu_{61} B+\alpha_{62} A b+\mu_{63} M P+\mu_{64} D C\right) \cdot T A A c \\
& \frac{d A B}{d t}=\alpha_{70} B-\left(\mu_{70}+\alpha_{71} C C+\alpha_{72} V C+\alpha_{73} T A A c+\alpha_{74} T A A v\right) \cdot A B
\end{aligned}
$$

Equation 4.1 describes Vaccine cells $(V C)$ dynamics. $V C$ are administered through an intraperitoneal injection according to a predefined dosage and vaccination schedule. The function $k_{i n}(t)$ models the inoculation and returns the number of vaccine cells inoculated into the host at time $t$ if a that time an injection is scheduled. As the vaccine cells come from an external event, this term represents the only source element in equation (4.1). Vaccine cells die for natural death $\left(\mu_{4}\right)$, are killed by Cytotoxic T cells $(T C)$ and Natural killer cells $(N K)$ which can kill vaccine cells coated by specific antibodies $(A B)$.

Tumor associated antigens released by vaccine cells $(T A A v)$ are proportional to the number of killed vaccine cells. This is the source element of the first part of equation (4.2). TAAv are subjected to natural degradation and phagocytosis by antigen presenting cells such as macrophages (MP), dendritic cells (DC) and $\mathrm{B}$ cells. $\mathrm{AB}$ can also bind to free $T A A v$ giving as a result immune complexes.

Activation of naive $M P$ and $D C$ (eqns. 4.3 and 4.4) mainly depends by $T A A v$ and $T A A c$ (tumor associated antigens released by $C C$ ). The first two terms of eqns. 4.3 and 4.4 are therefore used to estimate the ratio of newly activated $M P$ and $D C$ on the basis of $T A A v$ and $T A A c . M P$ and $D C$ can disappear $\left(-\mu_{17} M P\right.$ and $-\mu_{18} D C$ terms) due to natural death or due to the loss of their presentation status.

Equation (eqn. 4.5) describes plasma $B$ cells dynamics. $B$ can be stimulated to duplicate by the positive feedback of helper T cells $(T H)$. Interleukin $2(I L 2)$ plays an adjuvant role in this stimulation process. Death is modeled by $\mu_{20} B$ term.

$T H$ cells (eqn. 4.6) can be primed by major histocompatibility class II / peptide complex presentation through interactions with specialized antigen presenting cells. Presentation is not directly modeled, so 
the number of activated $T H$ is estimated on the number of $T A A v, T A A c$ and $V C$ in the system. Thanks to the ability of dendritic cells to cross-present allogeneic major histocompatibility class I (that is present in vaccine cells), vaccine cells actively contribute to the activation of helper $\mathrm{T}$ cells. Interleukin- 2 and Interleukin ( $I L 2$ and $I L 12$ ) also contribute to stimulate $T H$ cells duplication. Death is modeled by $-\mu_{21} T H$ term.

IL12 (eqn. 4.7) is introduced through the administration of the vaccine, so it depends on the dosage. Normal degradation is modeled by the term $-\mu_{5} I L 12$. IL12 is also partially absorbed for mitotic and stimulation signals by $T C$ and $T H$ priming and $N K$ activation. $I L 2$ stimulates $T H$ priming and primed $T H$ to further produce IL2. It is subjected to normal degradation $\left(-\mu_{30} I L 2\right)$ and it is partially absorbed for mitotic and stimulation signals in $T C$ priming and $B$ duplication.

In eqn. 4.9 the term $\left[\left(1-\frac{C C}{c_{\max }}\right)\right] \cdot\left[k_{1} C C\right]$ models $C C$ growth whereas the term $p_{1}$ is used to represent the continuous production of newborn cancer cells due to transgenic nature of the host. The other terms describe the death of $C C$ due to $N K, A B, T C$ actions.

Priming of Cytotoxic Cells (TC) (eqn. 4.10) mainly depends by $V C$ allogeneic major histocompatibility class I complex. Duplication is modeled by $\alpha_{41} T C$ term and normal death is modeled by $-\mu_{40} T C$ term.

$T A A c$ (eqn. 4.11) are proportional to the number of killed $C C$ and are subjected to degradation and phagocytosis by $B, M P$ and $D C$. $A B$ can also bind to free antigens producing immune complexes.

$A B$ (eqn. 4.12) are released by $B$ cells that differentiate into plasma $\mathrm{B}$ cells and are subjected to normal degradation (modeled by $-\mu_{70} A B$ term). Moreover they disappear binding to specific targets such as $C C, V C, T A A v$ and $T A A c$. NK are constant and do not vary during the experiment (e.g. $d / d t N K=0)$. Initial Cauchy conditions are set to 0 for all the equations except for NK whose initial value is set according the "in vivo" leukocyte formula .

A preliminary analysis of the model has been done [59]. In this analysis we presented the evolution of the system in a 3D states space using the $C C, T C$, and $A B$ variables curve, with the time representing the curves parameter. To this end the model has been solved numerically with Berkeley Madonna.

The untreated scenario has been initially analized. In this scenario the number of cancer cells grows with no control up to the saturation threshold, and the immune system is not able to contrast the tumor growth with the humoral (represented by $A B$ ) and cytotoxic (represented by $T C$ ) immune responses (figure 1(a)).

The second scenario reproduces the administration of the Early vaccination schedule, which consists in three vaccine cycles at the beginning of the experiment composed by two vaccine administrations over two weeks followed by two weeks of rest. The vaccine schedule is able to stimulate an initial immune response that constrasts the growth of tumor cells. This effect is represented by a large loop of the variables curve in the 3D states space due to the $T C$ and $A B$ actions. After that, cancer cells start to grow again with no constraints and the curve tends again towards the saturation threshold on the $C C$ axis (figure 1(b)).

The last scenario that has been considered is represented by the administration of the Chronic schedule. This schedule is composed by repeated Early cycle administrations for the entire life of the host. The administration of this schedule showed that, after an initial burst, cancer cells are eradicated and their level is kept low to a value near to 0 . The (immune system - cancer) system is stabilized and an equilibrium region is reached (figure $1(\mathrm{c})$ ).

This first analysis showed that the vaccine, when administered with a Chronic schedule, is able to stabilize the immune system - cancer competition around values that are safe for the host. Further refinements as well as asymptotic and sensitivity analyses and an analytical study of a simplified model are in progress and results will be presented in due course.

\section{Mathematical modeling of artificial immunity against melanoma}

Melanoma is an aggressive cancer and many immune system related therapies are presently under investigation. One of this is represented by adoptive transfer of activated $\mathrm{T}$ cells along with an immunological adjuvant. 


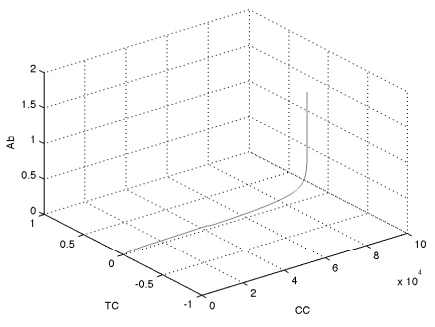

(a)

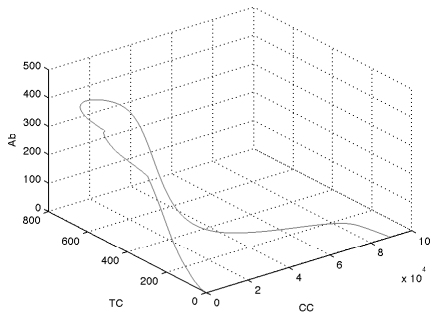

(b)

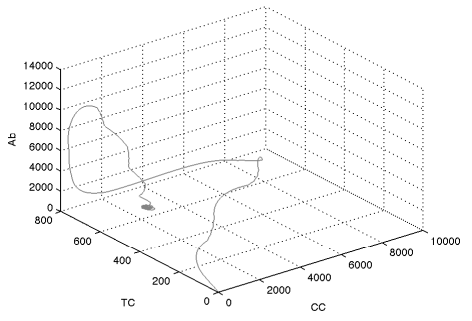

(c)

Figure 1. 3-D States' space for the untreated, Early and Chronic scenario

In order to deepen the study of the immune response stimulated by the administration of OT1 activated cytotoxic T cells with Anti-CD 137 immunostimulatory monoclonal antibodies against melanoma cells with the use of analytical tools, we built a preliminary ODE model developed to sketch the process. To catch-up the dynamics we considered in this case a two-compartments conceptual model and then we used Delay Difference Equations to model two different compartments: the injection point compartment where both antibodies and OT1 cells are injected, and the Skin compartment where melanoma develops. The preliminary system of equations has been presented in [60] and is shown as follows:

Injection point compartment

$$
\begin{aligned}
\frac{d E}{d t} & =k i n(t, p)-a_{11} E-a_{8} E \\
\frac{d A b}{d t} & =k i n(t, k)-a_{11} A b-a_{12} A b
\end{aligned}
$$

Skin compartment

$$
\begin{aligned}
\frac{d C}{d t} & =\left(a_{1}-a_{2} \ln (C)\right) \cdot C-a_{3} E_{s} C \\
\frac{d A}{d t} & =a_{4} \cdot\left(a_{3} C E_{s}\right)-a_{5} A \\
\frac{d E s}{d t} & =a_{7} A_{s} E_{s}+a_{11} E(t-\tau)+a_{6} N A-a_{8} E_{s} \\
\frac{d N}{d t} & =h(M-N)-a_{6} N A \\
\frac{d A_{s}}{d t} & =a_{11} A b(t-\tau)-a_{9} A_{s} E_{s}-a_{12} A_{s}
\end{aligned}
$$

The first two equations (4.13 and 4.14) model the first compartment and are used to reproduce the time evolution of both injected activated OT1 cytotoxic T cells $(E)$ and antibodies $(A b)$. kin $(t ; r)$ is a known function representing the number of inoculated entities $\mathrm{r}$ at the scheduled injection time $t$.

$E$ and $A b$ move to the skin where the melanoma develops (the second compartment) with given rates ( $-a_{11} E$ and $-a_{11} A b$ terms) and are subject to natural death or natural degradation ( $-a_{8} E$ and $-a_{12} A b$ terms). The melanoma cells $(C$, eqn. 4.15$)$ develop in the skin compartment. The term $\left(a_{1}-a_{2} \ln (C)\right) \cdot C$ represents a Gompertz growth and the second term models $C$ killing by Activated OT1 T cells that are in the skin $\left(E_{s}\right)$. Antigens $(A$, eqn. 4.16) are released in the skin compartment as a result of melanoma cells' killing by $E_{s}\left(a_{4} \cdot\left(a_{3} C E_{s}\right)\right)$ and are subject to natural degradation $\left(-a_{5} A\right)$.

The dynamics of Activated OT1 T cells in the skin compartment $\left(E_{s}\right)$ is described by equation 4.17. The term $a_{7} A_{s} E_{s}$ models the duplication of OT1 T cells. Anti CD-137 antibodies that have reached the skin compartment $\left(A_{s}\right)$ are able to boost OT1 $\mathrm{T}$ cells duplication. The term $a_{11} E(t-\tau)$ models the arrival in the skin compartment of injected OT1 $\mathrm{T}$ cells. These cells are supposed to be proportional to 
the number of OT1 $\mathrm{T}$ cells in the injection point compartment with a proportionality constant $a_{11}$ and a time delay of $\tau$. Moreover antigens released by killed melanoma cells may be captured by presenting cells such as macrophages and dendritic cells and presented to Naive cytotoxic T cells $(\mathrm{N})$ that may become active and then able to kill melanoma cells. This process is not actually modeled since it involves the inclusion of entities that are not fundamental at this first stage. An estimation of the newly activated OT1 cytotoxic $\mathrm{T}$ cells is then given by the term $a_{6} N A$. The term $-a_{8} E_{s}$ models the natural death of OT1 T cells.

Naive OT1 T cells that are already in the skin compartment are represented by eqn. 4.18. The term $h(M-N)$ is used to model homeostasis. $M$ is the number of circulating naive T cells under safe conditions given by the leukocyte formula. The second term $\left(a_{6} N A\right)$ represents the state change of cytotoxic $\mathrm{T}$ cells that go from naive to activated $\left(E_{s}\right)$.

Antibodies that have reached the skin compartment $\left(A_{s}\right.$, eqn. 4.19) are supposed to be proportional to the number of antibodies in the injection point compartment $(A b)$ with a proportionality constant $a_{11}$ and a time delay of $\tau$. They also stimulate OT1 cells activities and are subject to a natural degradation (terms $-a_{12} A_{s}$ and $-a_{9} A_{s} E_{s}$ ).

From a first analysis of the model we observed that the treatment acts in two ways: directly by activated OT1 cytotoxic T cells that are able to kill melanoma and Antibodies that boost T cells activities, and indirectly by promoting recruitment of naive OT1 cytotoxic T cells thanks to the releasing of melanoma cells antigens captured by presenting cells and then presented to these. Even in this case further improvement to the tuning as well as deeper analysis of the model are on the way and results will be presented in due course.

\section{Computational models}

Computational models use the language of computer science (including structures, algorithms and mathematical theories) to model a specific phenomenon. The most used method is represented by Cellular Automata $(\mathrm{CA})$, with all its variations and extensions. CA models are fully discretized dynamical systems that are well suited for computer simulations of biological systems [71]. The general framework for capturing dynamic behaviour in the models can be precisely tuned to mimic the behavior of the real system [40].

The initial idea of using a discrete automaton model in immunology was proposed by Kaufman et al. [25]. In this model, various cellular populations and interactions were represented by Boolean values. Several subsequent discrete models such as $[15,16,68,77]$ were all developed based on this idea. In [48], the Boolean structure was extended to a cellular automaton which adapted the concept of the "shape space" [78].

The automata that model complex systems or complex behavior are known as agent-based models or multi-agent systems. As in CA, there are rules governing interactive behavior and the agents "operate" in an environment of some sort. Many of the recent computational models of the immune system are agent based systems. The majority of current applications of simulation models are inspired by the early immunological CA proposed by Celada and Seiden [13] which attempted to build a general immune system simulator. Celada-Seiden model has provided useful insights into the dynamics of the immune system responses.

\subsection{Agent based models}

Since there is no simple mathematical description of Agent Based Models (ABM), there is only some real attempt to mathematically formalize simulators based on this approach [36]. The agent-based paradigm can be considered as an extension of the spin concept [43] where the wide-spread use of computers enabled the addition of more realistic descriptions of the internal state-space of the spins (the entities or agents) and the mathematical description of relatively complex interaction rules. There are only a few basic rules in the initial Celada-Seiden cellular automaton, while later developments extended and redefined 
the set of rules, resulting in improved generalization ability of the immune system cellular automaton. Their simulation program called IMMSIM has been used to investigate a number of immune system phenomena, for example affinity maturation and hypermutation of the humoral immune response [14]. The embedded rules can be extremely complex, as illustrated by the large number and complex expression of the Boolean rules developed in the Kaufman model [25]. More detailed reviews of CA and ABM can be found in $[21,39,61]$. In what follows, we would like to list several well-known examples of agent based models with special attention to tumor immunology modeling.

\section{Sim Triplex}

SimTriplex [52], one of the core simulator of the EC-funded ImmunoGrid project [23,54], is a specialized cellular automaton in modeling mammary carcinoma, Triplex vaccine and the immune system competition based on a modification of Celada-Seiden framework. It mimics the behavior of immune cells at the cellular level in both vaccinated and in naive mice. The simulation results have shown excellent agreement with in vivo experiments both for the time of formation of solid tumor and for the role of antibody responses in controlling tumor growth $[44,53]$. Once SimTriplex was validated, a new component was developed to optimize the Triplex vaccine protocol $[55,58]$ and help immunologists to find the best timing for administration of the vaccine [51].

To represent the tumor growth and the vaccine action, the following entities and interactions were modelled in SimTriplex: i) the cancer cells (CC) that encode their tumor associated antigens (TAA); they interact with antibodies (Ab), T-cells (TC) and natural killer cells (NK), ii) the vaccine cells (VC) that include TAAs, IL-12 and allogenic major histocompatibility molecules class I (MHCI); VC interact like CC, with $\mathrm{Ab}$, TC and NK, but the affinity function is modified by the presence of two adjuvants, iii) NK cells which, in the model, express only CD16a (Fc $\gamma$ RIIIA) receptor for ADCC mediated cytotoxicity; they interact with $\mathrm{Ab}$ coated $\mathrm{VC}$ and $\mathrm{CC}$. When two entities, which may interact, lie in the same lattice site then they interact following a probabilistic law. All entities which have capacity to mutually interact and are in the same site have a positive interaction.

\section{MetastaSim}

As previously said, the Triplex vaccine demonstrated able to almost prevent mammary carcinogenesis. After that, the therapeutic activity of the Triplex vaccine has been evaluated in BALBneuT mice against different stages of mammary carcinoma progression.

The study of the therapeutic activity showed that the vaccine loses progressively its efficacy with the advancement of tumor progression in BALBneuT mice, and has little or no efficacy at all against incipient mammary carcinomas. On the other hand, recent experiments showed that the Triplex vaccine can be an effective treatment against induced lung metastases induced in BALBneuT mice by intravenous injection of syngeneic mammary carcinoma cells [47].

From a translational point of view, this represents an important result. Phase-I clinical trials are in fact executed on advanced cancer patients to test toxicity and the presence of any undesirable effects. To this end a new model, hereafter referred as Metastasim [58], has been developed to optimize the efficacy of existing therapeutic treatments in order to speed-up the transfer of the treatment from mice to humans.

Metastasim can be considered as an extension of the SimTriplex model. However the two models differ in many ways and the differences are entitled with the biology, the location of the disease, and time-length that characterize the two in vivo experiments. The immunoprevention experiment lasts for 1 year whereas the therapeutic one lasts only for 1 month. SimTriplex uses a $16 \times 16$ lattice to simulate a small fraction of a mammary gland $\left(1 \mathrm{~mm}^{3}\right)$ whereas MetastaSim uses a $128 \times 128$ lattice to reproduce the frontal ventral surface of the left lung of a mouse, for an estimated volume of $64 \mathrm{~mm}^{3}$. Moreover cancer prevention is primarily driven by antibodies. Interferon- $\gamma(\operatorname{IFN}-\gamma)$ is the one of the major mediators both in cancer prevention and in the metastasis therapy whereas anti-HER-2/neu antibodies seem devoid of significant therapeutic activity. Other vaccine-induced mechanisms playing a causal role in the therapeutic experiment are represented by T-helper activities at the systemic level and macrophages infiltration in the tumor cell nests. 
In MetastaSim the the cancer growth kinetics has been totally rewritten in order to simulate multiple different metastatic nodules, each one with its own growth rate. The growth kinetics now uses the Gompertz growth law in its differential formulation, making the model an ABM - ODE hybrid one. The growth rates are randomly generated using as underlying distribution the cumulative distribution function $(\mathrm{CDF})$ coming from experimental measurements of the in vivo experiment (inverse transform sampling method). In this way the in silico nodules follow in time a gompertzian growth and their distribution in size at the end of the experiment resembles the in vivo one (in the sense that the two distributions cannot be considered statistically different).

Moreover a first simplified chemotaxis and some of the effects of the immunosuppression induced by cancer cells have been introduced into the model as well, in order to make the model more consistent with the last immunological advances.

In the in vivo experiment three sets of mice are used. All mice receive at day 0 an injection of metastatic cells. The first set (control set) is then treated with a placebo solution whereas the other two sets are treated respectively with Triplex +1 and Triplex +7 protocols. In the Triplex +1 protocol the administration of the vaccine starts one day after the intravenous injection of the metastatic cells and it is repeated twice weekly up to the end of the experiment (day 32 ). The Triplex +7 protocol uses the same twice-weekly cycle but started at day 7 . At the end of the experiment mice treated with Triplex +1 protocol showed a reduction of $99 \%$ of metastatic nodules (in respect to the untreated ones) and mice treated with Triplex +7 protocol showed a reduction of $\approx 82 \%$.

The model has been initially tuned in order to assess the value of free parameters using a random sample set of 8 virtual mice. It has then validated against existing in vivo experiments using two random sample sets of 100 virtual mice. None of the two sets contained mice coming from the tuning set. The in silico results over the two sample sets showed a good agreement with the in vivo results for all the reproduced scenarios (no vaccination, administration of the Triplex +1 protocol, administration of the Triplex +7 protocol) [58]. After that its first application has been the research of a protocol able to assure the same protection obtained using the Triplex +1 protocol but with fewer injections.

Wet biology requirements (i.e., the time required for the preparation of the vaccine) as well as the need to avoid undesirable effects entitle that no more than two vaccinations per week (in pre-established days) can be done. This means that for the length of the in vivo experiment (32 days) it is possible to administer the vaccine only in 9 days. The Triplex +1 protocol, which already counts 9 injections, is the most intensive one because it already uses all the available days to vaccinate. Shorter protocols should be therefore obtained by removing some injections from Triplex +1 protocol.

Using an exhaustive search over a search space of $2^{9}$ possible protocols, Metastasim showed that it is possible to obtain in silico a $45 \%$ reduction in the number of vaccinations. This reduction may represent an important result from the point of view of translational medicine, since a downsizing in the number of vaccinations is always advisable to minimize the risk of undesirable effects.

Moreover a further analysis of the results suggested that in order to deal with the exponential growth of cancer cells, any optimal protocol should be composed by an initial massive vaccine dosage followed by few vaccine recalls. Even if such kind of strategies are commonly used for many infectious diseases like tetanus and hepatitis-B, this suggestion can be considered as a new result in the field of cancer-vaccines immunotherapy.

\section{$\operatorname{SimB16}$}

Melanoma represents one of the most aggressive malignant tumors and it is due to the mutation of cells that produce the melanin (melanocytes). Many approaches in clinical and preclinical studies are actually based on the use and stimulation of cytotoxic $\mathrm{T}$ lymphocytes against melanoma cells. Immunological rejection of progressive tumors requires not only activation and expansion of tumor specific cytotoxic $\mathrm{T}$ lymphocytes (CTL), but also an efficient effector phase including migration of CTL in the tumor followed by conjugation and killing of target cells. 
SimB16 is an agent based model able to capture the general behavior of B16 melanoma and related immune system competition. We recently used this approach [56] to recapitulate both the effect of a specific immunotherapy strategy against B16-melanoma in mice and the tumor progression in a generic tissue section.

We performed 100 in silico experiments for each of the five treated cases plus 100 to compute statistics of the untreated case. A comparison of the in silico results with the in vivo experiments shows excellent agreement, suggesting the possible use of the simulator to predict new experimental results. Our results indicate that the agent-based model captures the essential components and dynamic of combined immunotherapy including adoptive $\mathrm{T}$ cell therapy and agonist anti-CD137 immunostimulatory monoclonal antibodies. The computer-assisted model predicts a critical role for CD137 expression on tumor vessel endothelium for successful therapy and other mechanistic aspects that will be experimentally addressed.

\section{Cancer immuno-therapies with C-ImmSim}

Vaccinology is often regarded as practical. Vaccines schedules are mainly empirical and based on the assumption that (sooner or later) an immune response can be generated and boosted by repetitive injections of antigens at times arbitrarily chosen. Vaccination protocols conceived to elicit an immune response against a malignancy have in most cases failed to achieve tumor eradication and/or in prolonging the survival of the patients.

Anti-cancer vaccination is based on the existence of antigens, selectively or preferentially expressed by tumors, called tumor-associated antigens (TAAs). These proteins do not generate an immune response, that is, they are not immunogenic, simply because they come from self-cells hence they are ignored by the immune defenses.

Most clinical trials contemplate multiple injections of one TAA (mainly in the form of protein, recombinant DNA or viruses modified to express the TAA) inoculated along with one highly immunogenic carrier. Carriers are typically derived from organisms that are phylogenetically very distant from the host.

Of the many thousands of peptides encoded by a complex antigen potentially presented to CD8 T cells, only a small fraction induces a non-negligible response in association with any given MHC class I allele, a phenomenon known as immunodominance [79]; an issue to be considered in designing optimal cytototoxic $\mathrm{T}$ cell mediated vaccination strategies

Finally, the crucial question is whether the use of multiple vectors has advantages over single carrier injections. Since a systematic comparison of the efficacy of different vaccination protocols is difficult due to technical limitations, we used a computational model to investigate the dynamics of the immune response to different anti-cancer vaccines based on randomly generated antigen/carrier compounds [10].

In order to evaluate the effects on both humoral and cellular immune response of repeated injections of TAA together with carriers, we used computer simulations based on a modified version of a well established computational model [13]. In particular, we compared protocols by using multiple different vectors/carriers and correlated the frequency of anti-tumor $\mathrm{T}$ cells and antibody titres with tumor control.

We have shown, how immune response to one given carrier does not necessarily favor immune response to weak antigens and after repetitive booster injections, anti-carrier can dominate over anti-tumor immune response [10].

Moreover, we have shown that injections at a 14 day-interval induce the highest average number of TAA-specific cytotoxic $\mathrm{T}$ cells and the best survival rate when combined with multiple carriers. Intriguingly, the difference in the results obtained with multiple carriers versus a single carrier is hampered when injections are delayed in time [10]. Probably the capability of vaccination to control the increased tumor mass is reduced, in this case, by the increased time delay before the immune response is mounted.

With regards to T cells, serotype-defined viruses that share most (if not all) T-helper and cytotoxic Tcell epitopes, do not induce strong cellular immune responses against each other. Actually, the opposite is true: the immune response to newly generated epitopes in the presence of strong (pre-existing) immunity is either strictly of primary kinetics [34] or even ineffective when immune system concentrates on dominant 
epitopes previously seen [30]. The explanation is that competition plays a pivotal role in shaping adaptive immune responses. Since resources in our body are limited and the number of antigens is enormous, this competition is instrumental to prevent redundant immune responses. In particular, competition is generated amongst circulating antibodies and dendritic cells to capture the injected TAA molecules; moreover competition develops almost invariably amongst different $\mathrm{T}$ cell clones.

Competition of $\mathrm{T}$ cells for space and growth factors has been suggested as a general mechanism $[5,27,73]$. However, it remains unclear how all these factors are, in turn, regulated [26,70]. In our model, competition amongst $\mathrm{T}$ cell clones for APC is caused by space limitation (mass effect) and by the killing of DCs by CD8 Ts.

Notably, experimental evidence shows that improvement of anti-TAA immunity has been obtained through removal of competing carrier epitopes [64]. Also, competition among CTLs has been demonstrated to limit the immune response [50], at least in specific experimental settings. Indeed, our model also suggests that circulating antibodies and dendritic cells compete to capture the injected molecules. This is in accordance with the finding that natural antibodies switch immune response from cellular to humoral [4].

\section{Conclusions}

Computational and mathematical modeling of the immune system is a busy research topic, but practical applications are still few. With the proofs of principle provided, particularly the demonstrated ability to accelerate discovery through simulation-driven experimentation and cyclical refinement of models, mathematical modeling of the immune system can now support practical applications. The use of hybrid models (i.e. ABM complemented with ODE models) could improve overall modeling power, taking advantage of detailed representation afforded by ABMs, computational tractability of ODEs, and, in particular, the ability to correct for body size. This correction will improve the usefulness of mathematical models studied in model organisms (such as mice) for the study of human immune responses. Nevertheless, the limitations of studies with model organisms for study of human pathologies could be taken into account.

The main disadvantage is that the models goodness strongly depends on available data and knowledge about the scenario that is going to be modeled. Moreover, in silico results must be experimentally verified.

The situation in which life scientists are unable to estimate a parameter or there is little or no knowledge about a specific process or scenario is quite usual. Theoretical models are, in these cases, able to focus on main mechanisms leaving out minor effects that can affect the represented scenario. Thus, they can support or contradict a theory or can be used to search for optimizing a desired experimental outcome.

Future perspectives of immune system modeling involve the acceleration of the developments of medical knowledge discovery and management, development of devices and procedures using in silico environment. Moreover it can be improved the interoperability of biomedical information and knowledge, providing patient-specific computer based models and simulation.

\section{References}

[1] A.K. Abbas, A.H. Lichtman, S. Pillai. Cellular and Molecular Immunology, 6th edn. (Elsevier, 2007).

[2] T. Alarcon, H.M. Byrne, P.K. Maini. A multiple scale model for tumor growth, SIAM. Multiscale Model Simul., 3, (2005) 440-475.

[3] R. Antia, C.T. Bergstrom, S.S. Pilyugin, S.M. Kaech, R. Ahmed. Models of CD8+ responses: 1. What is the antigenindependent proliferation program, J Theor Biol. 221, (2003) 585-598.

[4] V. Apostolopoulos, C. Osinski, I.F. McKenzie. MUC1 cross-reactive Gal alpha(1,3)Gal antibodies in humans switch immune responses from cellular to humoral, Nat. Med. 4, (1998) 315-320.

[5] T. Barthlott, G. Kassiotis, B. Stockinger. T cell regulation as a side effect of homeostasis and competition, J. Exp. Med. 4, (2003) 451-460.

[6] G.I. Bell. Mathematical Model of Clonal Selection and Antibody Production, Nature, 228, (1970) 739-744.

[7] N. Bellomo, M. Delitala. From the mathematical kinetic, and stochastic game theory to modelling mutations, onset, progression and immune competition of cancer cells, Physics of Life Reviews, 5, (2008) 183-206. 
[8] G.A. Bocharov. Modelling the Dynamics of LCMV Infection in Mice: Conventional and Exhaustive CTL Responses, J Theor Biol. 192(3), (1998) 283-308.

[9] K. Boggio, G. Nicoletti, E. Di Carlo, F. Cavallo, l. Landuzzi, C. Melani, M. Giovarelli, I. Rossi, P. Nanni, C. De Giovanni, P. Bouchard, S. Wolf, A. Modesti, P. Musiani, P.L. Lollini, M.P. Colombo, G. Forni. Interleukin 12-mediated prevention of spontaneous mammary adenocarcinomas in two lines of Her-2/neu transgenic mice, J Exp Med 188 (1998) 589-596.

[10] F. Castiglione, F. Toschi, M. Bernaschi, S. Succi, R. Benedetti, B. Falini, A. Liso. Computational modeling of the immune response to tumor antigens: implications for vaccination, J Theo Biol, 237(4) (2005) 390-400.

[11] F. Cavallo, R.A. Calogero, G. Forni. Are oncoantigens suitable targets for anti-tumour therapy?, Nat Rev Cancer 7 (2007) 707-713.

[12] F. Cavallo, C. De Giovanni, P. Nanni, G. Forni, P.L. Lollini. 2011: the immune hallmarks of cancer, Cancer Immunol Immunother 60 (2011) 319-326.

[13] F. Celada, P.E. Seiden. A computer model of cellular interaction in the immune system Immunol. Today 13, (1992) 56-62.

[14] F. Celada, P.E. Seiden. Affinity Maturation and Hypermutation in a Simulation of the Humoral Response, Eur J Immunol., 26, (1996) 1350-1358.

[15] D. Chowdhury, D. Stauffer, P.V. Choudary. A Unified Discrete Model of Immune Response, J Theor Biol. 145, (1990) 207-215.

[16] I.R. Cohen, H. Atlan. Network Regulation of Autoimmunity: An Automation Model, J Autoimmun., 2(5), (1989) 613-625.

[17] C. De Giovanni, G. Nicoletti, L. Landuzzi, A. Astolfi, S. Croci, A. Comes, S.Ferrini, R. Meazza, M. Iezzi, E. Di Carlo, P. Musiani, F.Cavallo, P. Nanni, P.L. Lollini. Immunoprevention of HER-2/neu transgenic mammary carcinoma through an interleukin 12-engineered allogeneic cell vaccine, Cancer Res 64 (2004) 4001-4009.

[18] G.P. Dunn, L.J. Old, R.D. Schreiber. The immunobiology of cancer immunosurveillance and immunoediting, Immunity 21 (2004) 137-148.

[19] S. Feyerabend, S. Stevanovic, C. Gouttefangeas, et al. Novel multi-peptide vaccination in Hla-A2+ hormone sensitive patients with biochemical relapse of prostate cancer, Prostate, 69(9), (2009) 917-927.

[20] O.J. Finn. Cancer immunology, N Engl J Med 358 (2008) 2704-2715.

[21] S. Forrest, Beauchemin. Computer Immunology, Immunol Rev. 216, (2007) 176-197.

[22] R.A. Gatenby, P.K. Maini, E.T. Gawlinski. Analysis of tumor as an inverse problem provides a novel theoretical framework for understanding tumor biology and therapy, Appl. Math. Letters, 15, (2002) 339-345.

[23] M. Halling-Brown, F. Pappalardo, N. Rapin, P. Zhang, et al. ImmunoGrid: Towards Agent-based Simulations of the Human Immune System at a Natural Scale, Philosophical Transactions A, 368, (2010) 2799-2815.

[24] M. Jílek, J. Stterzl. Model of Differentiation of Immunologically Competent Cell, in Developmental Aspects of Antibody Formation and Structure. (eds.), Academia, Prague, (1970) 963-981.

[25] M. Kaufman, J. Urbain, R. Thomas. Towards a Logical Analysis of the Immune Response, J Theor Biol. 114(4), (1985) 527-561.

[26] R.M. Kedl, J.W. Kappler, P. Marrack. Epitope dominance, competition and T cell affinity maturation, Curr. Opin. Immunol. 15 (2003), 120-127.

[27] R.M. Kedl, B.C. Schaefer, J.W. Kappler, P. Marrack. T cells down-modulate peptide-MHC complexes on APCs in vivo, Nat. Immunol. 3, (2002) 27-32.

[28] S. Kim-Schulze, B. Taback, H.L. Kaufman. Cytokine therapy for cancer, Surgical Oncology Clinics of North America, 16(4), (2007) 793-818.

[29] Z. Kirkali, E. T uzel.Systemic therapy of kidney cancer: tyrosine kinase inhibitors antiagiogenesis or IL-2?, Future Oncology, vol. 5(6), (2009) 871-888.

[30] P. Klenerman, R.M .Zinkernagel. Original antigenic sin impairs cytotoxic T lymphocyte responses to viruses bearing variant epitopes, Nature 394, (1998) 482-485.

[31] S. Koido, E. Hara, S. Homma, et al. Cancer vaccine by fusions of dendritic and cancer cells, Clinical and Developmental Immunology, 2009 (657369) (2009).

[32] C.A. Kruse, L. Cepeda, B. Owens, S.D. Johnson, J. Stears, K.O. Lillehei. Treatment of recurrent glioma with intracavitary alloreactive cytotoxic T lymphocytes and interleukin-2, Cancer Immunology Immunotherapy, 45(2), (1997) 77-87.

[33] Ha Youn Lee, D.J. Topham, Sung Yong Park, J. Hollenbaugh, et al.Simulation and Prediction of the Adaptive Immune Response to Influenza A Virus Infection, Journal of Virology, 83(14), (2009) 7151-7165.

[34] S. Liang, K. Mozdzanowska, G. Palladino, W. Gerhard. Heterosubtypic immunity to influenza type A virus in mice. Effector mechanisms and their longevity, J. Immunol. 152, (1994) 1653-1661.

[35] A. Lin, A. Schildknecht, L.T. Nguyen, P.S. Ohashi. Dendritic cells integrate signals from the tumor micro-environment to modulate immunity and tumor growth, Immunology Letters, 127(2), (2010) 77-84.

[36] J.F. Lynch. A Logical Characterization of Individual-Based Models, 23rd Annual IEEE Symposium on Logic in Computer Science, (2008) 379-390.

[37] P.-L. Lollini, F. Cavallo, P. Nanni, G. Forni. Vaccines for tumour prevention, Nat. Rev. Cancer, 6, (2006) $204-216$.

[38] P.L. Lollini, G. Nicoletti, L. Landuzzi, F. Cavallo, G. Forni, C. De Giovanni, P. Nanni. Vaccines and other immunological approaches for cancer immunoprevention, Curr Drug Targets (2010) Epub ahead of print.

[39] Y. Louzoun. The evolution of mathematical immunology, Immunological Reviews, 216, (2007) 9-20. 
[40] P. Manneville and Al. Cellular Automata and Modeling of Complex Physical Systems, Springer Verlag Series in Physics. 46, (1989).

[41] A. Mantovani, P. Allavena, A. Sica, F. Balkwill. Cancer- related inflammation, Nature, 454(7203), (2008) $436-444$.

[42] D.F. McDermott. Immunotherapy of metastatic renal cell carcinoma, Cancer, 115(10), (2009) 2298-2305.

[43] M. MezÂ' ard, G. Parisi, M. Virasoro. Spin Glass Theory and Beyond, World Scientific, Singapore (1988).

[44] S. Motta, F. Castiglione, P.-L. PLollini, F. Pappalardo. Modelling Vaccination Schedules for a Cancer Immunoprevention Vaccine, Immunome Research, 1:5, (2005) doi:10.1186/1745-7580-1-5.

[45] P. Nanni, G. Nicoletti, C. De Giovanni, L. Landuzzi, E. Di Carlo, F. Cavallo, S.M. Pupa, I. Rossi, M.P. Colombo, C. Ricci, A. Astolfi, P. Musiani, G. Forni, P.L. Lollini. Combined allogeneic tumor cell vaccination and systemi interleukin 12 prevents mammary carcinogenesis in HER-2/neu transgenic mice, J Exp Med 194 (2001) 1195-1205.

[46] P. Nanni, L. Landuzzi, G. Nicoletti, C. De Giovanni, I. Rossi, S. Croci, A. Astolfi, M. Iezzi, E. Di Carlo, P. Musiani, G. Forni, P.L. Lollini. Immunoprevention of mammary carcinoma in HER-2/neu transgenic mice is IFN-gamma and $B$ cell dependent, J Immunol 173 (2004) 2288-2296.

[47] P. Nanni, G. Nicoletti, A. Palladini, S. Croci, A. Murgo, A. Antognoli, L. Landuzzi, M. Fabbi, S. Ferrini, P. Musiani, M. Iezzi, C. De Giovanni, P.L. Lollini. Antimetastatic activity of a preventive cancer vaccine, Cancer Res., 67(22), (2007) 11037-11044.

[48] A.U. Neumann. Control of the Immune Response by a Threshold Automata Model on A Lattice, Physica A: Statistical Mechanics and Its Applications, 162, (1989) 1-19.

[49] L. Novellino, C. Castelli, G.Parmiani. A listing of human tumor antigens recognized by $T$ cells, Cancer Immunol Immunother 54 (2005) 187-207.

[50] M.J.Palmowski, E.M. Choi, I.F. Hermans, S.C. Gilbert, J.L. Chen, U. Gileadi, M. Salio, A. Van Pel, S. Man, E. Bonin, P. Liljestrom, P.R. Dunbar, V. Cerundolo. Competition between CTL narrows the immune response induced by prime-boost vaccination protocols, J Immunol. 168, (2002) 4391-4398.

[51] A. Palladini, G. Nicoletti, F. Pappalardo, A. Murgo, V. Grosso, V. Stivani, M.L. Ianzano, A. Antognoli, S. Croci, L. Landuzzi, C. De Giovanni, P. Nanni, S. Motta, P.-L. Lollini. In silico modeling and in vivo efficacy of cancer preventive vaccinations, Cancer Research, 70(20), (2010) 7755-7763.

[52] F. Pappalardo, F.Castiglione, P.-L. Lollini, S. Motta. Modelling and Simulation of Cancer Immunoprevention Vaccine, Bioinformatics, 21:12, (2005) 2891-2897.

[53] F. Pappalardo, S. Motta, P.-L. Lollini, E. Mastriani. Analysis of vaccine's schedules using models, Cellular Immunology, 244, (2006) 137-140.

[54] F. Pappalardo, M.D. Halling-Brown, N. Rapin, P. Zhang,et al. ImmunoGrid, an integrative environment for large-scale simulation of the immune system for vaccine discovery, design, and optimization, Briefings in Bioinformatics, 10:3, (2009) 330-340.

[55] F. Pappalardo, M. Pennisi, F. Castiglione, S. Motta. Vaccine protocols optimization: in silico experiences, Biotechnology Advances, 28, (2010) 82-93.

[56] F. 1 Pappalardo, I.M. Forero, M. Pennisi, A. Palazon, I. Melero, S. Motta. SimB16: modeling the combined anti-tumor effects of anti-CD137 monoclonal antibodies and adoptive $T$ cell therapy against a mouse melanoma model, BMC Cancer, submitted, (2011).

[57] C.R. Parish. Cancer immunotherapy: the past, the present and the future, Immunology and Cell Biology, 81(2), (2003) 106-113.

[58] M. Pennisi, F. Pappalardo, A. Palladini, G. Nicoletti, P.Nanni, P.-L. Lollini, S. Motta. Modeling the competition between lung metastases and the immune system using agents, BMC Bioinformatics, 11(Suppl 7):S13, (2010) doi:10.1186/14712105-11-S7-S13.

[59] M. Pennisi, C.Bianca, F. Pappalardo, S. Motta. Modeling artificial immunity against mammary carcinoma, Proceedings of the 10th International Conference on Mathematical Methods in Science and Engineering (CMMSE 2010), ISBN 97884-613-5510-5, (2010) 753-756.

[60] M. Pennisi, C. Bianca, F. Pappalardo, S. Motta. Compartmental mathematical modeling of immune system - melanoma competition, Proceedings of the 10th International Conference on Mathematical Methods in Science and Engineering (CMMSE 2011), ISBN 978-84-614-6167-7, (2011) 930-934.

[61] A.S. Perelson, G. Weisbuch. Immunology for physicists. Reviews of Moddern Physics, 69, (1997) $1219-1267$.

[62] A.M. Smith, A.S. Perelson. Influenza A virus infection kinetics: quantitative data and models, WIREs Syst Biol Med, 3(4), (2011) 429-445.

[63] H. Van Poppel, S. Joniau, S.W. Van Gool. Vaccine therapy in patients with renal cell carcinoma, European Urology, 55(6), (2009) 1333-1344.

[64] J. Rice, S. Buchan, F. Stevenson. Critical components of a DNA fusion vaccine able to induce protective cytotoxic T cells against a single epitope of a tumor antigen, J. Immunol. 169, (2002) 3908-3913.

[65] S.A. Rosenberg. Progress in human tumour immunology and immunotherapy, Nature, 411(6835), (2001) 380-384.

[66] S.A. Rosenberg, J.C. Yang, N.P. Restifo. Cancer immunotherapy: moving beyond current vaccines, Nat Med 10 (2004) 909-915.

[67] E. Sercarz, A.H. Coons. The Exhaustion of Specific Antibody Producing Capacity During A Secondary Response, In Mechanisms of Immunological Tolerance Conference. (eds.), Academia Prague, (1962) 78-83.

[68] H.B. Sieburg. A Logical Dynamic Systems Approach to the Regulation of Antigen-Driven Lymphocyte Stimulation, in Theoretical Immunology: Part I. A. S. Perelson (eds.), (1992) 273-293. 
[69] D.J. Slamon, W. Godolphin, L.A. Jones, J.A. Holt, S.G. Wong, D.E. Keith, W.J. Levin, S.G. Stuart, J. Udove, A. Ullrich. Studies of the HER-2/neu protooncogene in human breast and ovarian cancer, Science 244 (1989) 707-712.

[70] A.L. Smith, M.E Wikstrom, B. Fazekas de St Groth. Visualizing T cell competition for peptide/MHC complexes: a specific mechanism to minimize the effect of precursor frequency, Immunity 13, (2000) 783-794.

[71] D. Stauffer, R. Pandey. Immunologically Motivated Simulations of Cellular Automata, Computers in Physics. 6(4), (1992) 404

[72] J. Sterzl. Factors Determining the Differentiation Pathways of Immunocompetent Cells, Cold Spring Harb Symp Quant Biol. 32, (1967) 493-506.

[73] B. Stockinger, T. Barthlott, G. Kassiotis. T cell regulation: a special job or everyone's responsibility?, Nat. Immunol. $2,(2001) 757-758$.

[74] J. Ursini-Siegel, B. Schade, R.D. Cardiff, W.J. Muller. Insights from transgenic mouse models of ERBB2-induced breast cancer, Nat Rev Cancer 7 (2007) 389-397.

[75] K.E. de Visser, E. Eichten, L.M. Coussens. Paradoxical roles of the immune system during cancer development, Nature Reviews Cancer, 6(1), (2006) 24-37.

[76] L.M. Weiner, R. Surana, S. Wang. Monoclonal anti- bodies: versatile platforms for cancer immunotherapy, Nature Reviews Immunology, 10(5), (2010) 317-327.

[77] G. Weisbuch, H. Atlan. Control of the Immune Response, Journal of Physics A: Mathematical and General. 21, (1988) L189-L192.

[78] S. Wolfram. Theory and Applications of Cellular Automata, Redwood City, CA: Addison-Wesley (1986).

[79] J.W. Yewdell, J.R. Bennink. Immunodominance in major histocompatibility complex class I-restricted T lymphocyte responses, Annu. Rev. Immunol. 17, (1999) 51-88. 